\title{
On the Past, Present, and Future Role of Biology in NASA's Exploration of our Solar System
}

Dr. Kevin P. Hand, Jet Propulsion Laboratory, California Institute of Technology ${ }^{1}$, khand@jpl.nasa.gov, Planetary Science, Astrobiology [primary discipline(s)]

Dr. Cynthia B. Phillips, Jet Propulsion Laboratory, California Institute of Technology, Planetary Science Professor Christopher F. Chyba, Department of Astrophysics, Princeton University, Planetary Science, Astrobiology Professor Brandy Toner, University of Minnesota - Twin Cities, Biogeochemistry Dr. Kakani Katija, Monterey Bay Aquarium Research Institute, Bioengineering

Professor Victoria Orphan, California Institute of Technology, Environmental Microbiology, Geobiology Dr. Julie Huber, Woods Hole Oceanographic Institution, Marine Microbiology

Professor Colleen M. Cavanaugh, Dept. of Organismic and Evolutionary Biology, Harvard University, Microbial Ecology/Evolution and Symbiosis

Professor Marian Carlson, Director of Life Sciences, Simons Foundation, Professor Emerita of Genetics \& Development, Columbia University, Molecular Genetics

Professor Brent Christner, University of Florida, Microbiology

Professor Alexis Templeton, University of Colorado, Geobiology

Dr. Jeffrey Seewald, Woods Hole Oceanographic Institution, Geochemistry

Dr. Jason D. Hofgartner, Jet Propulsion Laboratory, California Institute of Technology, Planetary Science

Professor Jan P. Amend, Biological Sciences \& Earth Sciences Depts., Divisional Dean for Life Sciences, Univ. of Southern California, Environmental Microbiology, Geobiology

Dr. Beth N. Orcutt, Bigelow Laboratory for Ocean Sciences, Geomicrobiology

Professor Douglas H. Bartlett, Marine Biology Res. Div., Scripps Institution of Oceanography, Marine Microbiology

Professor Paul Falkowski, Environmental Biophysics and Molecular Ecology Program, Departments of Marine and

Coastal Sciences and Earth and Planetary Sciences, Rutgers University, Biological Oceanography

Rika Anderson, Assistant Professor of Biology, Carleton College, Northfield, MN, Geomicrobiology

Professor John R. Spear, Director, Quantitative Biosciences \& Engineering Program, Dept of Civil \& Env. Engineering, Colorado School of Mines, Microbiology

Dr. Tim Shank, Biology Department, Woods Hole Ocean Institution, Deep Sea Biology Professor Woodward W. Fischer, California Institute of Technology, Geobiology

Dr. Robert M. Hazen, Earth \& Planets Laboratory, Carnegie Institution for Science, Mineralogy, Planetary Evolution Dr. Tori Hoehler, NASA Ames Research Center, Biogeosciences

Professor Steven D'Hondt, Graduate School of Oceanography, University of Rhode Island, Oceanographer

Jo Pitesky, Jet Propulsion Laboratory, California Institute of Technology, Systems Engineering

Dr. Kennda Lynch, Lunar and Planetary Institute, USRA, Geomicrobiology, Astrobiology

Professor Everett L. Shock, Arizona State University, Geobiochemistry

Dr. Kate Craft, Applied Physics Laboratory, Johns Hopkins University, Planetary Science

Professor Eric Boyd, Montana State University, Microbiology

Professor Christopher H. House, Department of Geosciences and Earth and Environmental Sciences Institute, The Pennsylvania State University, Microbial Geochemistry

Professor Anna-Louise Reysenbach, Portland State University, Microbial Ecology

Professor Jennifer Glass, Georgia Institute of Technology, Geomicrobiology

Professor David Fike, Washington University in St. Louis, Biogeochemistry

Professor John A. Baross, School of Oceanography \& the Astrobiology Program, Univ. Washington, Microbiology, Oceanography

Professor Johann Peter Gogarten, University of Connecticut, Molecular and Cell Biology

Professor Betül Kaçar, Depts. of Astronomy; Molecular \& Cellular Biology; Lunar \& Planetary Laboratory, University of Arizona, Evolutionary Biology and Paleogenetics

Professor Moh El-Naggar, University of Southern California, Physics, Biological Sciences, and Chemistry Depts., Biological Physics

\footnotetext{
1 This work was carried out at the Jet Propulsion Laboratory, California Institute of Technology, under a contract with the National Aeronautics and Space Administration (80NM0018D0004). 
Professor Alison E. Murray, Division of Earth and Ecosystem Sciences, Desert Research Institute, Microbial Ecology \& Biological Oceanography

Professor Christopher Dupont, J. Craig Venter Institute, Genomics and Engineering Biology Dr. Jennifer Scully, Jet Propulsion Laboratory, California Institute of Technology, Planetary Science Dr. Lynn Rothschild, NASA Ames Research Center, Synthetic Biology, Astrobiology

Professor Elizabeth Trembath-Reichert, School of Earth \& Space Exp., Arizona State University, Geomicrobiology, Astrobiology

Dr. Frieder Klein, Woods Hole Oceanographic Institution, Fluid-Rock Interactions Professor Phoebe A. Cohen, Williams College, Paleobiology

Professor Gillian H. Gile, Arizona State University, Evolutionary Microbiology

Professor Karen Lloyd, University of Tennessee, Department of microbiology, Geomicrobiology Professor Anne Dekas, Stanford University, Geomicrobiology

Professor John R. Delaney, School of Oceanography, University of Washington, Submarine volcano-hydrothermal Systems

Professor Mark Skidmore, Montana State University, Geomicrobiology

Dr. Joy Buongiorno, Earth and Planets Laboratory, Carnegie Institution for Science, Microbiologist

Professor Karyn Rogers, Rensselaer Polytechnic Institute, Extremophile Microbiology, Prebiotic Chemistry

Dr. Amy Hofmann, Jet Propulsion Laboratory, California Institute of Technology, Planetary Science Professor William J. Brazelton, University of Utah, Microbiology

Professor Ariel Anbar, School of Earth \& Space Exp., School of Molecular Science, Arizona State University,

Biogeochemistry

Dana Ann Manalang, Applied Physics Lab, University of Washington, Ocean Instrumentation

Professor Bradley Stevenson, Dept. of Microbiology and Plant Biology, University of Oklahoma, Microbiology

Professor Susanne Neuer, Arizona State University, Biological Oceanography

Ellie Hara, University of Colorado-Boulder, PhD student, Geochemistry

Dr. Thomas Nordheim, Jet Propulsion Laboratory, California Institute of Technology, Planetary Science

Professor Russell Shapiro, Dept. of Geological \& Environmental Sci., California State University, Chico, Geobiology

Professor Alexander S. Bradley, Washington University in St. Louis, Biogeochemistry

Professor Jill Mikucki, University of Tennessee, Microbial Ecology

Professor Michael E. Brown, California Institute of Technology, Astronomy, Planetary Science

Professor Mihaela Glamoclija, Department of Earth \& Environmental Sciences, Rutgers University, Geomicrobiology

Dr. Carolina Reyes, University of Vienna, Dept. of Environmental Geoscience, Microbiology, Geomicrobiology

Professor Mónica Sánchez-Román, Vrije Univ. Amsterdam, The Netherlands, Geomicrobiology and Geochemistry

Professor Jack D Farmer, Arizona State University, Paleobiology

Professor Donato Giovannelli, Department of Biology, University of Naples Federico II, Microbiologist

Professor Gurol Suel, UC San Diego, Molecular Biology

Samantha Trumbo, California Institute of Technology, PhD Student, Astronomy, Planetary Science

Dr. Marissa Cameron, Jet Propulsion Laboratory, California Institute of Technology, Planetary Science

Professor Magdalena Osburn, Northwestern University, Geobiology

Dr. James Andrew Bradley, Queen Mary University of London, Geobiology

Professor Ferran Garcia-Pichel, School of Life Sciences, Arizona State University, Microbiomics

Professor Andrew D. Steen, Departments of Microbiology and Earth and Planetary Sciences, University of Tennessee,

Geomicrobiology

Professor Jeffrey Marlow, Boston University, Marine Microbiology, Geobiology

Dr. Gareth Trubl, Lawrence Livermore National Lab, Soil Virology

Dr. Kirtland Robinson, Woods Hole Oceanographic Institution, Aqueous Organic Geochemistry

Tristan Caro, Department of Geological Sciences, University of Colorado Boulder. PhD student, Microbiology

Victoria Fulfer, University of Rhode Island, PhD student, Oceanography

Dr. Ceth W. Parker, Jet Propulsion Laboratory, Caltech, Planetary Protection

Jayme Feyhl-Buska, University of Southern California, PhD student, Microbiology

Anaïs Roussel, Georgetown University, PhD Student, Geobiology, Astrobiology 


\section{Executive Summary}

Here we provide a brief, but we hope useful, perspective on the role of biology in NASA's planetary science goals, and its spacecraft missions, past, present, and future. We argue that while biology - via astrobiology - generates much interest and excitement for NASA, biology is vastly under-represented as a science within NASA Planetary Science Division missions.

Our key points detailed in this paper are as follows:

1. Biology, as a science and fundamental discipline, is not adequately represented within NASA's Planetary Science Division and mission portfolio.

2. The search for life beyond Earth should include a search for biosignatures on worlds that could host extant life, thus providing the potential to truly understand a biochemistry distinct and independent from that of life on Earth.

3. We know how to search for signs of life. Decades of advances in biology, chemistry, geology, geobiology, and instrumentation have yielded a robust framework for searching for signs of life, be it extinct or extant.

4. We know where to go to search for extant life: the ocean worlds of the outer solar system, and the subsurface of Mars offer the best locales. In particular, Europa and Enceladus provide the best places to search for extant water- and carbon-based life that has potentially arisen independent of life on Earth. The top Flagship mission priority for the next decade should be to land on the surface of Europa or Enceladus to look for signs of life in the ice, and to directly study the chemistry, geology, and geophysics of the ice shell and subsurface ocean. This exploration would directly address a long-standing question of "Does life exist beyond Earth, and do organisms live there now?" We note that the direct investigation of putative plumes on Europa will be made possible by the Europa Clipper mission, and plumes on Enceladus could be sampled by a New Frontiers class mission. We recommend continued support for Clipper, and the potential to propose Enceladus missions to New Frontiers.

5. The in situ surface exploration of Europa and Enceladus will ignite a new age of ocean exploration and catalyze a new field of research: comparative oceanography. Oceans, much like planetary atmospheres over the past many decades, will become an attribute and process that extends to worlds beyond Earth; studying ocean worlds can help us better understand oceans as widescale planetary phenomena. Such work will enable new discoveries in oceanography, while also helping to inform us about how Earth's ocean works, and how to protect it.

6. The next decade of solar system exploration could be the decade in which life beyond Earth is discovered within our solar system. We know where to go, and how to search; now we just need the will to pursue a dedicated program of exploration. Four hundred years after Galileo revolutionized our understanding of our place in the universe, we may once again be on the verge of a scientific revolution, this time in biology.

\section{Biology within NASA's Planetary Science Division}

Humanity has been exploring our solar system with robotic spacecraft for a little over 57 years (Mariner 2, launched in August of 1962, made the first planetary flyby - of Venus - in December of that year). In the years and decades since, planetary science and astrobiology have grown and expanded, both in terms of exploration targets and science questions. Here we provide a brief, but we hope useful, perspective on the role of biology in NASA's planetary science goals and spacecraft missions, past, present, and future. We argue that while biology - via astrobiology - 
generates much interest and excitement for NASA, biology is vastly under-represented as a science within NASA spacecraft missions.

While astrobiology and planetary science can and should be seen as distinct, they clearly overlap and there is often confusion as to where one ends and the other begins. Definitions for planetary science are varied, but usually reflect its multidisciplinary nature. The 2011 Vision and Voyages Decadal Survey for Solar System Exploration (Space Studies Board (2011), hereafter denoted by SSB2011) focused on planetary science and defined it as follows:

"Planetary science is shorthand for the broad array of scientific disciplines that collectively seek answers to basic questions such as how do planets form, how do they work, and why is at least one planet the abode of life. These basic motivations explain why planetary science is an important undertaking, worthy of public support."

Here the boldface is ours and is used to draw attention to two key aspects of this definition. While the 'array of scientific disciplines' is never defined, one could argue that the most basic disciplines from which to form an array would be: astronomy, physics, chemistry, geology, and biology. In addition, the second bolded clause specifically implicates biology as one of the necessary disciplines in the array, since surely biology would be important - perhaps the most important when seeking answers to the question 'why is at least one planet the abode of life.'

After defining 'planetary science', SSB2011 then proceeds to argue for programmatic balance across mission types - Discovery, New Frontiers, and Flagship (please see page S-5 of SSB2011 for detail). While programmatic balance sounds good and is useful from a cost and implementation perspective, such balance did not consider the array of fundamental scientific disciplines and how they are incorporated into different mission types. We argue that programmatic balance should also incorporate the role of the basic scientific disciplines within missions, that is, the roles should be balanced. Doing so is important both for the fundamental nature of the science and scientific questions, but also for inclusivity: more scientific disciplines means a greater diversity of expertise, backgrounds, and ideas. These are central tenets to NASA and are part of what guides NASA's scientific vision and workforce (NASA, 2020).

Next we consider the array of disciplines that comprise planetary science, and examine the role of each discipline throughout NASA's history of solar system exploration. We consider physics, chemistry, geology, and biology to be the four most fundamental fields of science, and assess each mission according to the fields of science to which they are most responsive. Figure $\mathbf{1}$ provides an overview of that assessment for Flagship, New Frontiers, and Discovery class missions. While these assessment and checkmarks are somewhat subjective, they are relatively straightforward and obvious.

As can be seen in the matrix below, the disciplines of physics, chemistry, and geology have been well served by NASA's current exploration and science strategy. Biology, however, is not well-represented. If we examine the programmatic balance aspect of mission types, we see that this difference is further exacerbated, as biology has really only played a role in a few Flagshipclass missions. Recently, the selection of the Dragonfly mission as part of the New Frontiers portfolio represents a significant step forward for the inclusion of biology in solar system exploration. As a whole, however, solar system exploration has been exclusive and limited to investigations that predominantly address physics. Biology, and the search for life, meanwhile, have served as much of the attraction for public engagement and to justify solar system exploration.

Part of the explanation for the bias excluding biology is that a certain level of scientific reconnaissance needs to occur before we understand whether or not questions of a biological nature could be applicable to any given target. This made sense for the initial stages of our robotic exploration of the solar system, as the physics, chemistry, and geology of a world lay the foundation for life's origins, and planetary habitability. Moving forward, however, we argue that biology should 


\begin{tabular}{|c|c|c|c|c|c|c|c|c|c|c|c|}
\hline $\begin{array}{l}\text { Flagship } \\
\text { Missions }\end{array}$ & $\begin{array}{l}\text { 음 } \\
\text { 형 } \\
\frac{0}{0} \\
\frac{0}{2}\end{array}$ & $\begin{array}{l}\text { 둥 } \\
\frac{5}{6} \\
\frac{0}{0} \\
\frac{0}{2}\end{array}$ & క. & 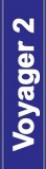 & 竞 & $\begin{array}{l}\text { N } \\
\frac{0}{5} \\
\frac{1}{5}\end{array}$ & 递 & 章 & 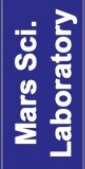 & 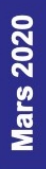 & 究 \\
\hline Physics & $x$ & $x$ & $x$ & $x$ & $x$ & $x$ & $x$ & $x$ & $x$ & $x$ & $x$ \\
\hline Geology & & & $x$ & $x$ & $x$ & $x$ & $x$ & $x$ & $x$ & $x$ & $x$ \\
\hline Chemistry & $x$ & $x$ & $x$ & $x$ & $x$ & $x$ & $x$ & $x$ & $x$ & $x$ & $x$ \\
\hline Biology & & & & & $x$ & $x$ & & & & I & \\
\hline
\end{tabular}

\begin{tabular}{|c|c|c|c|c|}
\hline $\begin{array}{l}\text { New } \\
\text { Frontiers } \\
\text { Missions }\end{array}$ & 궁 & $\stackrel{\circ}{\text { 옥 }}$ & 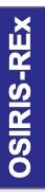 & 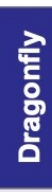 \\
\hline Physics & $x$ & $x$ & $x$ & $x$ \\
\hline Geology & $x$ & & $x$ & $x$ \\
\hline Chemistry & $x$ & $x$ & $x$ & $x$ \\
\hline Biology & & & & $x$ \\
\hline
\end{tabular}

\begin{tabular}{|c|c|c|c|c|c|c|c|c|c|c|c|c|c|c|c|c|}
\hline $\begin{array}{l}\text { Discovery } \\
\text { Missions }\end{array}$ & 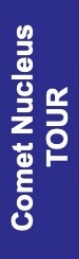 & $\frac{0}{\frac{0}{6}}$ & 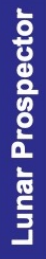 & 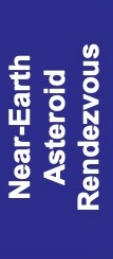 & 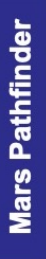 & 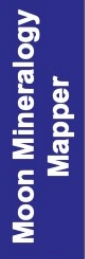 & $\frac{\frac{5}{0}}{\frac{0}{0}}$ & 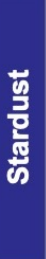 & $\frac{1}{\frac{1}{c}}$ & 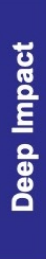 & 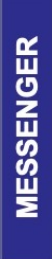 & 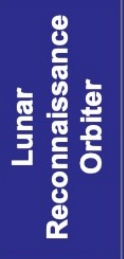 & 恣 & $\begin{array}{l}\text { 봉 } \\
\frac{0}{0} \\
\text { E }\end{array}$ & उे & $\frac{\frac{9}{0}}{0}$ \\
\hline Physics & $x$ & $x$ & $x$ & $x$ & & & $x$ & $x$ & $x$ & & $x$ & $x$ & $x$ & $x$ & $x$ & $x$ \\
\hline Geology & $x$ & & $x$ & $x$ & $x$ & $x$ & & & $x$ & $x$ & $x$ & $x$ & $x$ & $x$ & $x$ & $x$ \\
\hline Chemistry & $x$ & $x$ & $x$ & $x$ & $x$ & $x$ & & $x$ & & $x$ & I & $x$ & $x$ & & $x$ & $x$ \\
\hline Biology & & & & & & & & & & & & & & & & \\
\hline
\end{tabular}

Figure 1. As defined by SSB2011, planetary science is shorthand for the broad array of scientific disciplines that collectively seek answers to basic questions such as how do planets form, how do they work, and why is at least one planet the abode for life. Shown above are most of NASA's planetary missions, and as can be seen, biology has not been well represented as a discipline, despite being central to one of the grandest questions NASA seeks to answer: Is there life beyond Earth? (NASA, 2020).

play an ever-increasing role in our exploration strategy. We do not need to assess the habitability of every world before making progress on the specific habitability of one or two worlds. In addition, we know enough about at least two worlds in the outer solar system - Europa and Enceladus - to warrant a direct in situ search for biosignatures on the surfaces of these worlds.

\section{Extant versus Extinct Life}

The distinction between the search for extant and extinct life is critical if we are to understand biology as a potentially universal process. Extant life is, of course, life that is alive and exists today; extinct life no longer exists, but it may have left behind fossils or other signs of its past existence. The search for extant life enables us to investigate at least two key questions: 1) Does this life represent a second, independent origin of life? and 2) How does its biochemistry work in service to Darwinian evolution (e.g., information storage, mutation, and replication)?

The large biomolecules of life - such as DNA, RNA, and proteins - do not last long as 'fossils.' (Consider the dramatic example of dinosaur bones: no definitive long strands of DNA have ever been recovered from even the most well-preserved of fossils. The same is true for ancient microbial life on Earth - the large biomolecules do not survive for much longer than a few to a few $\sim 10$ 's of millions of years in the rock record.) The search for extant life prioritizes exploring environments where life could be alive today, and in which we might begin to understand how that life works and what its relation is - if any - to life on Earth. Does it contain DNA or does it function on some other large biomolecules for information storage, replication, and repair? Are there many separate 'trees of life' within our solar system, or is the tree of life on Earth the only 
one? The search for past life on worlds like Mars is very important, but the search for extant life is how we will truly revolutionize biology (if life exists beyond Earth).

SSB2011 captured some of the importance of this issue in its Planetary Habitats theme statement: "Beyond Earth, are there contemporary habitats elsewhere in the solar system with necessary conditions, organic matter, water, energy, and nutrients to sustain life, and do organisms live there now?"

We advocate for an exploration strategy that targets environments where life could be alive today, such as beneath the icy shells of Ocean Worlds, or deep within the subsurface of Mars. As detailed in the next section, the search for extant life still employs many of the same measurements and tools used in the search for signs of extinct life, but the ultimate goal is to find an organism that can be studied at the biochemical level.

\section{We know how to search for signs of life}

Biology as a science has experienced tremendous advances since the launch of Mariner 2 in 1962. As Figure 2 illustrates, the discovery of DNA was less than a decade old, and that discovery would later give rise to the polymerase chain reaction (PCR) and other genetic and proteomic techniques that would revolutionize our understanding of life on Earth.

Meanwhile, the Viking Landers set down in 1976 and their payloads were designed based on the best techniques available at that time. This meant that many of the methods focused on culturing viable microbes; many of the advanced analytical chemistry and genetic techniques were not yet available. Significantly, it was not until 1977 when the discoveries of Archaea, hydrothermal vents, and cryptoendoliths in Antarctic rocks would further transform our understanding of the extremes of life on Earth.

In the decades since Viking, biologists, chemists, geologists, and geobiologists, have greatly advanced our understanding of biology's fingerprint in ancient rocks on Earth, and what

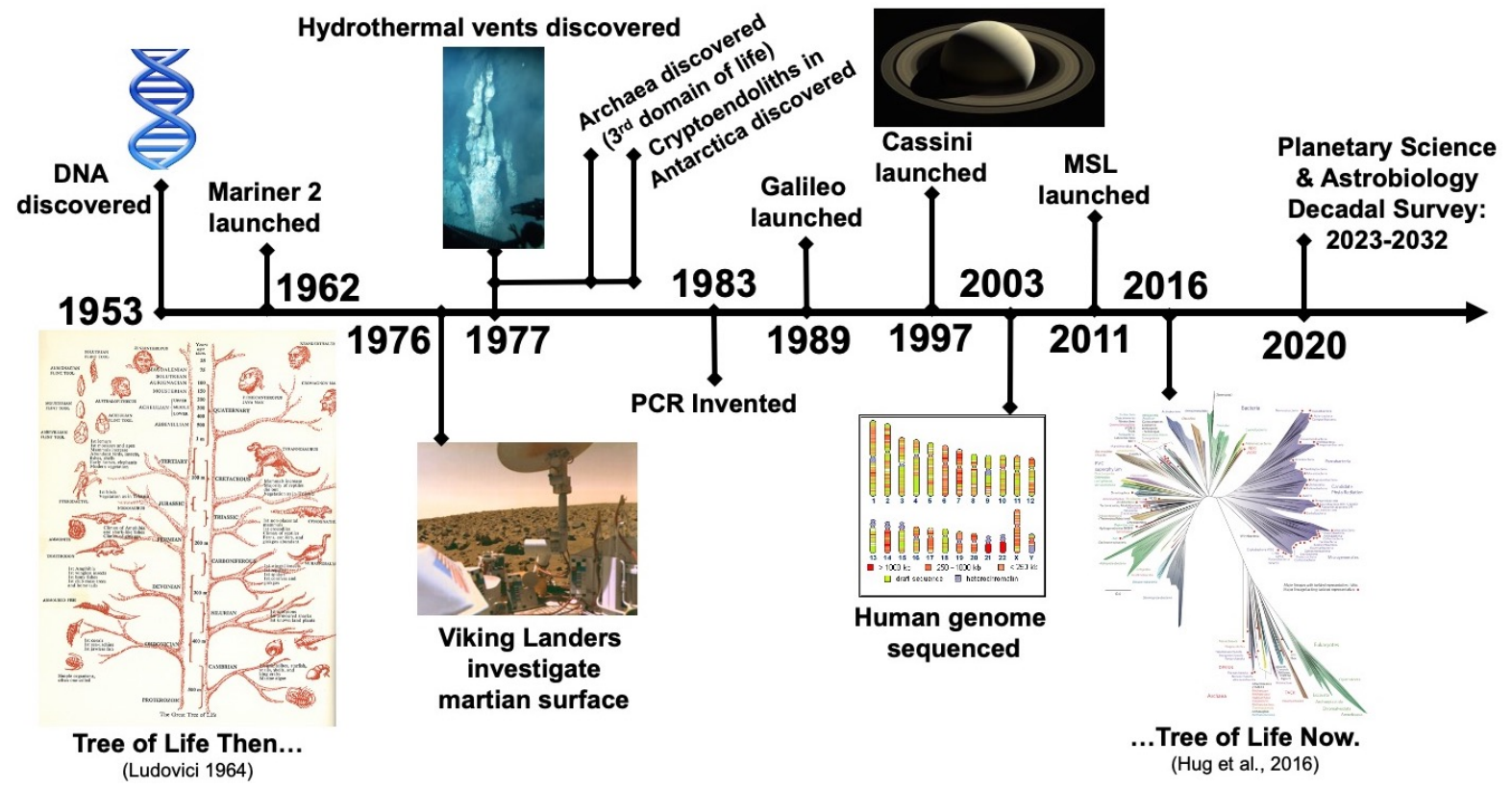

Figure 2. The science of biology has come a long way since the middle of the last century. The last time NASA truly engaged in a direct search for biosignatures was with the Viking Landers. Those missions occurred long before many key developments in biology, many of which have helped inform our framework for biosignatures. We are ready to directly search for signs of life on the surfaces of ocean worlds, and in the martian subsurface. 
measurements (and measurement combinations) are needed to provide a robust approach to seeking signs of life - whether it be in ancient rocks on Earth or in rocks and ices on distant worlds. Many lessons have been learned from our exploration of Mars (Chyba and Phillips, 2001) and the framework that was developed for Mars has broad applicability across the solar system (Mustard et al., 2013).

We now know how to search for signs of life, extant and extinct, in the rock record of Mars or within the icy surface of an ocean world. As detailed in the Space Studies and National Research Council 2002 report on 'Signs of Life', the five key classes of measurements that should be prioritized are: 1) Morphology, 2) Organic Chemistry \& Biochemistry, 3) Inorganic Chemistry, 4) Isotopic Analyses, and 5) Environmental Measurements. Also detailed in that report are candidate instruments for conducting the measurements.

Coupled with measurements must be an overarching strategy, which was well-summarized in Chyba and Phillips (2001) and details five essential elements: 1) If the payload permits, conduct experiments that assume contrasting definitions for life, 2) Given limited payload, the biochemical definition of life deserves priority, 3) Establishing the geological and chemical context of the environment is critical, 4) Life-detection experiments should provide valuable information regardless of the biology results, and lastly, 5) Exploration need not, and often cannot, be hypothesis testing. Planetary missions are often missions of exploration; and therefore, the above guidelines must be put in the context of exploration and discovery driven science.

In the nearly 45 years since Viking, and the two decades since many of the reports and papers cited above, the scientific community has continued to study the topic of biosignatures and how to ensure a robust framework for assessing biosignatures and life detection. That work has reinforced many of the findings from decades ago and has provided a strong and rigorous foundation from which to now engage in a direct search for signs of life in our solar system (please see links to the Europa Lander SDT Report and NASA's Life Detection Ladder in the references).

\section{We know where to go}

The best targets in the search for life beyond Earth, and in particular the search for extant life, are the ocean worlds of the outer solar system and, potentially, the deep subsurface of Mars. One limiting factor for Mars is that if we to discover DNA-based life on Mars, it might be indicative of life that has travelled between Earth and Mars, not a separate, independent origin of life. The ocean worlds of the outer solar system, however, are much harder to 'seed' with Earth life, and thus DNA-based life on those worlds could be indicative of convergent evolution leading to two independent trees of life, both utilizing DNA.

With this in mind, we prioritize Europa and Enceladus as the premier targets in the search for signs of life. Titan is an additionally fascinating world, and the Dragonfly mission will make pioneering measurements of the physical, chemical, geological, and possibly biological processes on that world.

Europa and Enceladus both harbor chemically-rich subsurface liquid water oceans that may be habitable for life as we know it, and which may have given rise to an independent origin of life. There are a number of scientific differences to consider when prioritizing one over the other, but either world presents an incredible opportunity for biology. Landing on the surface of Europa or Enceladus to conduct a direct in situ search for signs of life is our top priority for the coming decade. Importantly, NASA has committed to the Europa Clipper mission, which will return data that can be used to select a landing site for a future lander. The plumes of Enceladus lend themselves to a New Frontiers class flyby mission that searches for signs of life in the plumes and carries reconnaissance instrumentation for a follow-on lander. Critically, investments made into 
landing on Europa also feed-forward into landing on Enceladus and other airless icy moons (please see the link to mission concept resources in the references).

We note that there are some in the planetary science community who argue that before a pathfinding astrobiology lander can go to the surface of an ocean world, we must survey all of the candidate ocean worlds, and then complete landing reconnaissance of them before committing to a lander. This approach would likely yield much useful planetary science, but it would not significantly advance biology and the search for life. By analogy, it would be akin to requiring one to map out all of the rainforests on planet Earth before ever stepping foot into one. We are ready to take the next step and directly explore the surface of Europa or Enceladus.

\section{Comparative oceanography and a new age of ocean exploration}

Europa and Enceladus are the top targets for advancing biology, but they are equally compelling targets for oceanography and opening a new window into oceans as widescale planetary processes. In much the same way that James Lovelock's pioneering research on atmospheres was influenced by contemplating the atmospheres of Venus, Earth, and Mars (Lovelock, 1979), the direct exploration of Europa and Enceladus could initiate a new field of comparative oceanography. Here again, getting down to the surfaces of Europa and Enceladus to conduct detailed analyses of surface material, and of the surface and subsurface environment, is the top priority for the coming decade. Not only does such work create new fields and help inform our understanding of Earth's ocean, but it also engages a more diverse scientific community and grows the NASA community.

\section{This could be the decade of discovery}

Over four hundred years ago, Galileo put the final nail in the coffin of Aristotelian cosmology, advancing what became known as the Copernican revolution. The wandering orbs were shown to be worlds like our own, orbiting the Sun, harboring mountains and moons. The Sun was a star, and stars were Suns. The Earth was a planet, and some planets might be like Earth. Planetary science was born.

In the decades and centuries that followed we would come to appreciate that the laws of physics work beyond Earth. With the advent of spectroscopy, we would recognize that the principles of chemistry also work beyond Earth. And with the advent of the space age investigation of the Moon, Venus, Mercury, and Mars, we would learn that geology also works beyond Earth.

But when it comes to biology, we have yet to make that leap. We do not yet know whether or not biology, the phenomenon of life, works beyond Earth. We of course know that we can bring life into space beyond Earth, and it works, but we do not know if life, as a process, works independent of life on Earth, and serves as a fundamental process within our Universe.

Now, as our robotic exploration of space begins its seventh decade, we can pursue this civilization-scale question. For the first time in the history of humanity we have the tools and technology capable of directly measuring signs of life on other worlds.

In summary, we prioritize taking the next logical step in pursuit of answering the question of whether or not biology is a universal process and exists beyond Earth: landing on the surface of Europa or Enceladus to search for signs of life, and to directly study an ocean world, should be NASA's top Flagship mission priority for the coming decade. NASA has committed to Europa Clipper, which will make it possible to select a landing site on Europa. The most logical next step is to send a lander in close cadence with Clipper. Such a mission would advance the science of biology, as well as physics, chemistry, and geology. 


\section{$\underline{\text { References }}$}

Space Studies Board (2011). Vision and Voyages for Planetary Science in the Decade 2013-2022.

Washington, D.C.: The National Academies Press. doi:10.17226/13117.

NASA (2020) https://science.nasa.gov/about-us/science-strategy

Chyba, C. F., \& Phillips, C. B. (2001). Possible ecosystems and the search for life on Europa. Proceedings of the National Academy of Sciences, 98(3), 801-804.

Mustard, J. F., Adler, M., Allwood, A., Bass, D. S., Beaty, D. W., Bell, J. F., ... \& Edgett, K. S. (2013). Report of the Mars 2020 science definition team. Mars Explor. Progr. Anal. Gr, 150, 1-154.

Space Studies Board and National Research Council (2002). Signs of life: A report based on the April 2000 Workshop on Life Detection Techniques. National Academies Press.

Europa Lander Science Definition Team Report: https://europa.nasa.gov/resources/58/europalander-study-2016-report/

NASA Life Detection Ladder: https://astrobiology.nasa.gov/research/life-detection/ladder/

Europa Lander Mission Concept Resources: https://www.jpl.nasa.gov/missions/europa-lander/

Lovelock, J. E. (1979). Gaia: a new look at life on Earth. Oxford: Oxford University Press.

Hug, L. A., Baker, B. J., Anantharaman, K., Brown, C. T., Probst, A. J., Castelle, C. J., ... \& Suzuki, Y. (2016). A new view of the tree of life. Nature microbiology, 1(5), 1-6.

Ludovici, L.J., (1964) The Great Tree of Life. Aldine Press, J.M. Dent \& Sons Ltd., Phoenix House, Bedford St. London. 